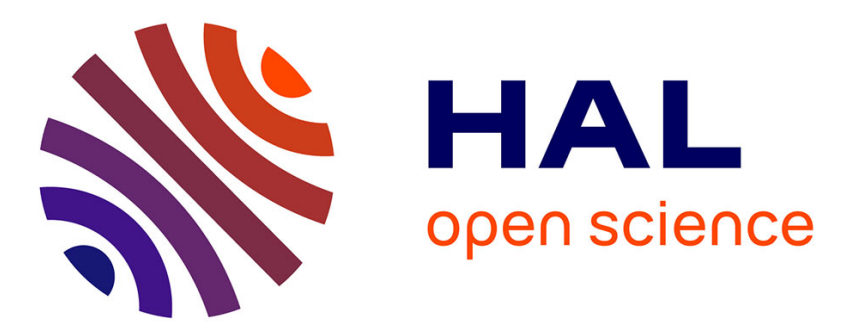

\title{
La révolution nationale et l'exaltation impériale à la réunion durant la période de Vichy (1940-1942) \\ Pierre-Éric Fageol
}

\section{To cite this version:}

Pierre-Éric Fageol. La révolution nationale et l'exaltation impériale à la réunion durant la période de Vichy (1940-1942). Guerres mondiales et conflits contemporains, 2012, 246, pp.41-62. 10.3917/gmcc.246.0041 . hal-01244088

\section{HAL Id: hal-01244088 \\ https://hal.univ-reunion.fr/hal-01244088}

Submitted on 11 Feb 2022

HAL is a multi-disciplinary open access archive for the deposit and dissemination of scientific research documents, whether they are published or not. The documents may come from teaching and research institutions in France or abroad, or from public or private research centers.
L'archive ouverte pluridisciplinaire HAL, est destinée au dépôt et à la diffusion de documents scientifiques de niveau recherche, publiés ou non, émanant des établissements d'enseignement et de recherche français ou étrangers, des laboratoires publics ou privés. 


\title{
LA RÉVOLUTION NATIONALE ET L'EXALTATION IMPÉRIALE À LA RÉUNION DURANT LA PÉRIODE DE VICHY (1940-1942)
}

\begin{abstract}
" Notre devoir est simple, à nous Français d'outre-mer, deux fois Français, liés indissolublement à la patrie aujourd'hui malheureuse ; nous sommes avec elle dans la mauvaise fortune comme nous l'avons été dans des temps meilleurs. Nous participerons avec tous nos moyens, aux efforts du Gouvernement français dans l'œuvre de rénovation nationale qu'il a courageusement entreprise et qui porte déjà ses fruits. " ${ }^{1}$
\end{abstract}

C'est par ces mots que le gouverneur de La Réunion, Pierre Aubert, répond aux interrogations suscitées au sein de l'opinion publique réunionnaise par l'entrevue de Montoire en octobre 1940. Le positionnement de l'île sur la suite à donner aux événements est dans tous les esprits, et nul ne songe à déroger à la règle de la légalité politique et du dévouement patriotique à la mère patrie. Cependant, par le prisme de la Révolution nationale et des nouvelles valeurs défendues par le régime de Vichy, le principe patriotique est en rupture totale avec le passé. Loin de s'inscrire dans un patriotisme révolutionnaire où la patrie n'est qu'une réalité idéologique, les discours mettent plutôt en valeur un patriotisme " traditionnel » qui fait de la France un être moral doté de vertus dont l'incarnation est composée des éléments constitutifs de la société. Si pour la première forme, l'impulsion ne peut provenir que de la métropole en qualité de principe identitaire sublimé ; pour la seconde, elle doit être issue de la réalité nationale et donc des valeurs défendues par le pays. C'est pourquoi la mise en évidence des vertus de la colonie au sein d'un Empire glorifié devient un leitmotiv dans les discours de la période considérée.

Selon cette logique, la notion de centre et de périphérie s'en trouve perturbée. L'initiative appartient désormais à la périphérie coloniale. La Révolution nationale se double ainsi d'une révolution impériale permettant une fusion identitaire entre le "national » et le "colonial ». Peu importe la réalité politique d'une telle rhétorique. Même si l'Empire n'est plus qu'une peau de chagrin ${ }^{2}$ et de plus en plus coupé des réalités métropolitaines, les

1. Archives départementales de La Réunion (ADR) 1M4003, Discours Pierre Aubert, 31 octobre 1940.

2. Jean-Pierre Azéma et François Bédarida (éds.), Vichy et les Français, Paris, Fayard, 1992, p. 122-134. 
discours idéalisent une conception impériale régénératrice de la nation. Le mythe colonial de Vichy met ainsi en évidence que «moins il y avait d'empire réel, plus il y avait d'empire idéalisé »3.

Dans ce contexte, il est délicat de se soustraire de la logique officielle prônée par les autorités coloniales de l'île qui ont créé un artefact impérial d'autant plus mobilisateur qu'il s'exerce hors de toute pression de l'occupant. Le principe d'autorité s'applique dès lors sans fard et dans toute sa rigueur ${ }^{4}$ sur une société réunionnaise, certes sceptique sur la marche à suivre, mais qui peut se reconnaitre dans certaines valeurs ainsi défendues. Au-delà d'une divergence d'intérêt ou d'un réel enracinement social du régime, il convient surtout de s'interroger sur la force persuasive d'une telle rhétorique.

\section{LES ENJEUX D’UN POSITIONNEMENT POLITIQUE}

\section{Le temps des choix}

L'adhésion des autorités coloniales au pétainisme s'inscrit dans une logique historique de maintien de la légalité politique mais s'explique également par la spécificité du contexte réunionnais depuis la déclaration de la guerre. La prise de fonction dès le début de l'année 1940 du gouverneur Pierre Aubert rassure d'abord l'opinion publique soucieuse, depuis le départ à la retraite de son prédécesseur Joseph Court le 3 décembre 1939, de continuer l'effort de guerre alors consenti. En effet, sa carrière plaide en faveur de l'enthousiasme qu'il suscite dès son arrivée sur l'île comme en témoignent les déclarations dans la presse des édiles locaux. Les très aimables paroles de bienvenue dont il a été honoré à son arrivée lui ont été droit au cœur, rappelant ainsi que «la perle de l'océan Indien a revêtu, une fois de plus, pour recevoir son gouverneur, cette parure de "gentillesse" qui fleure la vieille province française $\|^{5}$. Sa carrière honorable suit les méandres des réseaux coloniaux où l'allégeance personnelle fait souvent figure de carte de visite. La proximité de destin entre les différents administrateurs de l'Empire explique pour une bonne part les choix entrepris par chacun lors de la défaite de la France. Le mimétisme de contrainte explique ainsi les prises de position parfois contradictoires avec les idéaux initialement déclarés par le gouverneur Pierre Aubert. En effet, l'analyse de ses discours entre février et juin 1940 témoigne d'une volte-face politique qui l'amène dans un premier temps à défendre le principe d'une participation active à l'effort de guerre avant d'accepter les conséquences de l'armistice.

3. Pascal Blanchard et Ruth Ginio, "Révolution impériale : le mythe colonial de Vichy (19401944), in Pascal Blanchard, Sandrine Lemaire et Nicolas Bancel (éds.), Culture coloniale en France. De la Révolution française à nos jours, Paris, CNRs Éd., 2008, p. 376.

4. Eric Jennings, «La politique coloniale de Vichy », in Jacques Cantier et Eric Jennings, L'Empire colonial sous Vichy, Paris, Odile Jacob, 2004, p. 8.

5. ADR 1M4003, Discours Pierre Aubert, 27 février 1940. 
Dès son intronisation, Pierre Aubert fait preuve d'une certaine prudence en faisant en sorte que "La Réunion, tout en sacrifiant largement à l'autel de la patrie, non seulement souffre le moins possible de l'état de guerre, mais poursuive encore la route de son développement harmonieux $»^{6}$. Ce n'est qu'avec le début des hostilités sur le sol français et les premiers remaniements politiques qui s'en suivent que le gouverneur s'implique plus résolument dans une propagande patriotique très largement éculée. Annonçant le 26 mai 1940 le remaniement ministériel qui voit le retour du maréchal Pétain au sein du Gouvernement, il n'hésite pas à exprimer toute sa confiance dans la victoire finale que laissent préjuger les atouts dont dispose encore la France pour faire face à l'ennemi.

"Ces hommes, ces noms, ces chefs, notre magnifique armée [...], les armées alliées et les magnifiques exploits des aviateurs [...], un Gouvernement dynamique et fort, nous forgent une cuirasse de confiance en la Victoire finale. Il est interdit de douter. Le civil n'est plus un spectateur, tantôt plein d'espoir, tantôt angoissé ; le combat est économique autant que militaire " ${ }^{7}$.

Le principe de la guerre totale et l'injonction de la victoire sont ainsi mis en exergue. Cette dernière ne sera que le fruit d'un effort conjugué des forces armées, de la cohésion politique et des concessions économiques consenties par les Français. Dans cette logorrhée où les superlatifs sont légion, la nation en armes peut également compter sur l'appui des Britanniques et notamment sur leur potentiel impérial qui est capable de "mobiliser des forces formidables qui emporteront la décision " ${ }^{8}$. Surtout, la victoire semble inéluctable, car les forces déployées sont « au service d'un idéal : le respect de la personne humaine ». Cette allocution radiodiffusée fit une très forte impression sur tous ceux qui l'entendirent et recueillit selon la presse l'adhésion unanime de la population réunionnaise 9 . Les paroles du chef de la colonie sont celles que l'opinion attendait ${ }^{10}$, "elles disent ce qu'elles doivent dire $"^{11}$ et ne souffrent d'aucun commentaire. Elles prouvent le courage "d'un homme du Nord envahi $»^{12}$, ayant subi les ravages de la soldatesque allemande. C'est pourquoi « les Réunionnais ont trop le sentiment de leur responsabilité morale envers la patrie pour ne pas se sentir entièrement d'accord avec leur gouverneur $~{ }^{13}$.

Lorsque l'épilogue tragique de la bataille de France est connu, le gouverneur s'empresse de rassurer ses administrés en rappelant que « de toutes les parties du Monde sont parvenus les vœux ardents des Français d'outre-mer et l'expression de leur volonté de sacrifier au redressement de la patrie

6. Ibid.

7. ADR 1M4003, Allocution radiodiffusée, 26 mai 1940.

8. Ibid.

9. Le Progrès, 27 mai 1940.

10. Le Peuple, 28 mai 1940

11. Le Travail, 28 mai 1940.

12. Né le 8 mars 1888, Pierre Aubert est issu de la petite bourgeoisie arrageoise

13. Le Peuple, 28 mai 1940. 
blessée ${ }^{14}$. La presse met ainsi en parallèle à la fois le discours de Pierre Aubert et les premières bribes d'informations émanant d'une France Libre encore balbutiante. Informant l'opinion qu'un certain "Général de Gol » (sic) s'est imposé la noble tâche de constituer un comité d'union nationale français, les journaux divulguent les appels réitérés diffusés sur la BBC qui s'en prennent à la capitulation du Gouvernement Pétain. L'Empire n'est pas resté insensible à ces appels et « d'après les dernières nouvelles reçues de Londres, le mouvement du Général de Gol progresse rapidement en dehors de la France ${ }^{15}$. N'ayant pas reçu d'instructions de la part de son ministère de référence, Pierre Aubert se montre prudent quant au positionnement que cela implique pour le gouvernorat, même si les discours prononcés depuis son arrivée sur l'île laissent entrevoir un ralliement à la France Libre dont les idéaux rejoignent en partie ceux qu'il professe. D'ailleurs, ne proclame-t-il pas au gouverneur de Madagascar Marcel de Coppet sa «foi ardente en une victoire finale qu'obtiendront les Alliés en poursuivant en commun la guerre avec l'appui poussé de leurs empires ${ }^{16}$. Cette parfaite communion d'âme avec le gouverneur de Madagascar peut être interprétée comme un acte de subordination ou comme la résultante d'un certain pragmatisme politique. La Réunion dépendant militairement de la Grande Ile, les enjeux stratégiques impliquaient un tel rapprochement ${ }^{17}$.

De la même manière, Pierre Aubert s'appuie sur les velléités patriotiques exprimées par les corps constituants de l'île plutôt favorables à poursuivre le combat. Même s'il tempère ce qu'il considère comme des manifestations inopportunes, il relaie auprès de sa hiérarchie les messages de soutien à la métropole et les avis favorables à la poursuite des combats ${ }^{18}$.

Pourtant, même si les prises de position sont plutôt propices à la sécession, le ralliement au régime de Vichy semble inévitable. Ce revirement politique s'explique à la fois par des considérations personnelles et par la prise en compte des enjeux politiques propres à la colonie. Les liens personnels entretenus par Pierre Aubert avec le gouverneur de Madagascar Léon Cayla ont certainement influencé sa décision. Nouvellement nommé à la tête de la Grande Île en remplacement de Marcel de Coppet, jugé trop favorable à la poursuite des combats, ce dernier se positionne immédiatement en faveur du Maréchal. Pierre Aubert sait désormais qu'il risque de perdre son poste en cas de décision contraire. Les injonctions du nouveau régime en place à Vichy et la volonté de ne pas déroger à son devoir d'administrateur colonial ont assurément pesé d'un grand poids dans sa décision finale. Cependant, d'autres facteurs entrent en ligne de compte. L'anglophobie latente de nombreux administrateurs coloniaux a pu inconsciemment repousser l'idée d'un rapprochement avec les Britanniques. C'est

14. ADR 1M4003, Discours Pierre Aubert, 24 juin 1940.

15. Le Progrès, 25 juin 1940.

16. ADR 1M4068, Télégramme au gouverneur de Madagascar, non daté. (1941).

17. Archives nationales de l'Outre-mer (АNOM) 1Affpol 1131, Rapport politique Pierre Aubert

18. АNOM 1 Affpol 796, Allocution radiodiffusée, 21 juin 1940. 
d'autant plus probant que les contentieux impériaux s'inscrivent dans une réalité propre à l'océan Indien. Or le 23 juin, Pierre Aubert reçoit la visite de Maurice Gaud, consul britannique à La Réunion, qui lui remet de la part du sous-secrétaire d'État britannique des Affaires étrangères le message suivant :

«Le présent Gouvernement français en acceptant sous la contrainte les conditions de l'ennemi pour un armistice a été empêché de remplir la garantie solennelle de la France envers ses alliés britanniques. [...] Nous, le Gouvernement britannique et le Peuple britannique sommes résolus à continuer la lutte jusqu'à la fin. Nous avons fait appel aux autorités civiles et militaires de tous les territoires français d'outre-mer de se battre la main dans la main avec nous jusqu'à la victoire et ainsi remplir la garantie de la République française. Nous ferons tout en notre pouvoir pour maintenir l'intégrité et la stabilité économique de tous les territoires français d'outre-mer. Nous garantissons de plus que ces territoires seront pourvus de fonds pour couvrir le paiement des salaires et pensions de tous les officiers civils et militaires dans tout l'Empire français d'outre-mer qui sont prêts à coopérer avec nous. " ${ }^{19}$

Au-delà du ton comminatoire de ce message, sont définis les enjeux de la légalité supposée du nouveau régime et par ce biais la validité de l'action du gouverneur. De même que les administrateurs coloniaux français puissent dépendre de la mansuétude britannique peut être considéré par Pierre Aubert comme une situation intolérable. Ce dernier ayant reçu l'ordre de ne prendre aucune décision précipitée, il se contente d'être évasif dans sa réponse et se pare d'une douce attitude diplomatique. Maurice Gaud entreprend alors de diffuser sur les ondes cette injonction britannique et de mettre ainsi l'opinion publique au fait des tractations entreprises. Ce choix est perçu comme une ingérence abusive puisque Pierre Aubert considère que "certaines affaires qui touchent au Gouvernement ne se traitent pas sur la place publique $»^{20}$. Ayant le souci de protéger ses prérogatives, il entend alors restaurer son pouvoir et annihiler les oppositions qui se font jour sur la scène publique. Les ardeurs patriotiques jusqu'alors exprimées doivent désormais s'inscrire dans « une obéissance disciplinée au chef responsable par un labeur silencieux et soutenu, par la plus grande réserve dans les propos $»^{21}$. Il estime alors nécessaire de convoquer la Commission consultative de la défense le 29 juin où il expose les enjeux liés au maintien de la légalité politique et les interrogations suscitées par la demande britannique.

\section{Les raisons d'un positionnement}

Lors de cette réunion, il annonce qu'en se positionnant en dehors de la légalité politique cela entraînerait la nomination d'un nouveau chef pour

19. ANOM. 1Affpol 796, Message du sous-secrétaire d'État britannique, 23 juin 1940.

20. ANOM 1Affpol 796, Allocution radiodiffusée, 25 juin 1940.

21. ANOM 1Affpol 796, Allocution radiodiffusée, 24 juin 1940. 
la colonie que la métropole imposerait unilatéralement. Surtout, en supposant une quelconque rébellion, il craint que cela n'entraîne des représailles à la fois de la part du Gouvernement métropolitain mais aussi des autorités militaires allemandes. Il apparaît donc que l'intérêt de La Réunion n'est pas de "se ranger en enfant perdu aux côtés de l'Angleterre " ${ }^{22}$. Surtout, Pierre Aubert rappelle que "La Réunion occupe sur la carte du monde une place infiniment modeste qui lui impose de jouer le rôle de satellite de la Grande Île malgache ${ }^{23}$. Or, cette dernière ayant fait acte d'allégeance en faveur de Vichy, La Réunion ne dispose d'aucune latitude pour entreprendre une action isolée. Enfin, Pierre Aubert se targue de défendre une certaine éthique professionnelle et n'entend pas quitter son poste dans la honte puis récuse l'idée de devenir un "mercenaire susceptible de passer aux gages d'une autre puissance $"^{24}$. Selon ses propres termes, ses trente-deux années de loyaux services lui donnent l'intime conviction qu'il ne peut se délier du serment de fidélité à son pays. Fort de cette argumentation, il soumet ensuite à la sagacité des membres de la commission consultative de la défense la question suivante :

«Le Gouvernement de La Réunion doit-il sortir de la légalité, au mépris des ordres du pouvoir central, à la disposition du Gouvernement britannique ? "25

Les différents membres sont invités à s'exprimer à tour de rôle et récusent la formulation abrupte proposée par le gouverneur. En dépit des doutes sur la légalité du gouvernement de Bordeaux et sur son véritable poids politique dans une France aux trois quarts occupée, la foi en la victoire et l'espoir suscité par l'aide britannique transpirent dans l'ensemble des interventions. Le président du Conseil général, Raoul Nativel, exprime ainsi ses doutes sur les enjeux stratégiques d'une telle perspective :

«La question qui se pose, à mon avis, n'est pas de savoir si l'île de La Réunion doit se mettre en rébellion contre le Gouvernement français. Cette question ne peut pas, en l'état de nos informations, être posée. Elle le pourrait si l'Administration le pouvoir élu de ce pays avaient la certitude que le Gouvernement de Bordeaux est un Gouvernement libre. $»^{26}$

Ce postulat étant établi, il reprend ensuite son argumentation en offrant des perspectives plus globales sur les conditions du conflit :

«Nous, Empire, intact à tous points de vue, partie intégrante de l'Empire français, rien de nous autorise [...] à tenir pour un fait définitivement acquis, la défaite de la France métropolitaine, qui est la seule broyée. $»^{27}$

C'est ainsi que la France impériale, partie intégrante de la plus grande France n'est pas sous la botte allemande et dispose des moyens nécessaires

22. ANOM 1Affpol 796, Rapport politique de Pierre Aubert (1940).

23. Ibid.

24. Ibid.

25. Ibid.

26. Ibid.

27. Ibid. 
pour continuer le conflit. Or, seule l'Angleterre est à même de pouvoir soutenir l'effort souhaité par les territoires de l'Empire. Selon ses propres termes, il ne s'agit aucunement de s'inféoder à un pays étranger et " de tourner le dos à la France ${ }^{28}$. Si les autres membres de la Commission sont plus timorés dans leurs interventions, ils s'inscrivent néanmoins dans la même logique. C'est ainsi que le président de la Commission coloniale, M. Lagourgue, estime le maintien des alliances comme une question d'honneur :

«Aujourd'hui, parce que la France est envahie, nous tournons le dos à nos alliés, et donnons à nos ennemis, nos armes, nos camions, nos avions, nos tanks ; et cela, pour aider à combattre une amie avec laquelle nous marchions la main dans la main. Dans ces conditions, je trouve que la proposition faite par l'Angleterre est magnanime. $»^{29}$

Peu à peu, le gouverneur reçoit pourtant le soutien de certains membres de la Commission et impose une ligne de conduite légaliste qui ne souffre aucune critique. Les membres sont ainsi rappelés à l'ordre, et les menaces à peine voilées les confrontent aux incertitudes de leurs devoirs administratifs. La ligne politique est clairement établie et nul ne doute d'un ralliement in fine à Vichy. Somme toute, c'est sans aucun doute le docteur Raymond Vergès qui résume le mieux la situation en distinguant les devoirs propres aux fonctionnaires et ceux qui s'attachent à la personne humaine. En qualité de fonctionnaire, le soutien au gouverneur semble s'imposer à l'instar d'une pesanteur déontologique coloniale. Néanmoins, en tant qu'homme, il lui est pénible de voir "la France foulée aux pieds" et préconise une continuation de la lutte "aux côtés de n'importe qui pour le salut de la France ${ }^{30}$. Chimère désormais dépassée dans le cadre d'une administration coloniale de plus en plus coercitive. Les discours de Pierre Aubert délimiteront ainsi progressivement un champ politique sous haute surveillance dont le seul principe est « de faire respecter l'ordre et la tranquillité publique $»^{31}$.

Le gouverneur est d'autant plus conforté dans son action que les pouvoirs et les prérogatives du nouveau chef de l'État français sont officialisés dans la colonie le 22 juillet. Il se fait fort également de rappeler que les deux députés réunionnais Lucien Gasparin et Auguste Brunet ont voté sans scrupule et sans consultation de leur électorat les pleins pouvoirs à Pétain. Une symbiose est dorénavant établie entre les représentants du peuple et ceux de l'État. La Réunion s'enlise alors dans une sourde complaisance envers le nouveau régime, et les quelques velléités belliqueuses exprimées lors de la signature de l'armistice se dispersent dans les cénacles clandestins. Toute la force politique de Pierre Aubert est d'avoir su tempérer les ardeurs patriotiques en reconnaissant leur légitimité tout en clamant leur caducité.

28. Ibid.

29. Ibid.

30. Ibid.

31. ADR 1M4003, Discours Pierre Aubert, 10 août 1940. 
Jamais le sentiment d'appartenance nationale ne fut aussi éloigné de son principe de représentation.

Les premières mesures remettant en cause les fondements démocratiques ne font qu'entériner par la suite le principe autoritaire ainsi défini. Dès octobre 1940, Pierre Aubert entreprend une réforme des institutions locales. Le Conseil général est dorénavant dissous et remplacé par une Commission administrative restreinte. De même, les maires ainsi que les conseillers municipaux sont soumis à l'approbation des autorités de Vichy. Le Conseil privé est épuré de ses membres anglophiles et secondé d'un Conseil économique consultatif favorable au nouveau régime. Dans ce nouveau cadre, la représentation politique de l'île se trouve amoindrie. En sonnant le glas de la République, le régime de Vichy a en quelque sorte " recolonisé " ses anciennes possessions et remis en cause le processus historique d'émancipation des sociétés coloniales. Cette perspective est perçue a posteriori, non sans cynisme par le gouverneur : "Le crédit sympathique rencontré par les initiatives du Gouvernement central [...] marque assez la fragilité et le caractère artificiel de l'ancienne organisation, dans un pays, où une administration tutélaire doit guider, surveiller, protéger une population où les éléments évolués sont en minorité. "32 $^{32}$

Ces propos témoignent d'un profond mépris envers le processus d'assimilation qu'il considère comme inadapté au contexte réunionnais puisque les élections n'ont jamais entériné "une représentation réelle des différents éléments de la population $»^{33}$. Pour distingués que soient la plupart des mandataires réunionnais, il pense qu'ils ne représentent guère qu'euxmêmes ou bien les intérêts particuliers dont ils restent les porte-parole. Cette scission "entre le pays réel et sa représentation élue " ${ }^{34}$ expliquerait l'impopularité du régime défunt et l'espoir suscité par la Révolution nationale.

Au-delà des considérations politiques qui expliquent la prise de position en faveur de Vichy, on peut admettre une concordance de principes idéologiques entre les administrateurs coloniaux et les idéaux défendus par le nouveau régime. Le zèle déployé pour mettre en place les principes de la Révolution nationale en constitue certainement la meilleure preuve. L'idée que la France puisse être sauvée par son Empire ne fait que renforcer le sens du devoir des autorités coloniales désormais investies d'une mission salvatrice. Pourtant, cette dernière ne peut s'extraire des réalités sociales et des mentalités qui la sous-tendent. Comment mobiliser les masses sans prendre en compte leurs attentes et leurs craintes, qu'elles soient réelles ou imaginaires? Cette condition est d'autant plus nécessaire que la réalité de la Révolution nationale s'inscrit dans un microcosme ayant ses propres modalités d'interprétation.

\footnotetext{
32. Rapport politique Pierre Aubert (1941), op. cit.

33. Ibid.

34. Ibid.
} 


\section{Une opinion en proie aux doutes}

Malgré une indéniable popularité des cérémonies orchestrées par le nouveau régime, les interrogations demeurent quant à la sincérité de l'élan populaire mis en exergue par la propagande. S'agit-il d'une volonté des apparatchiks du gouvernorat provichyste et de leurs séides ou de la véritable marque d'un engouement général ? Le danger serait de fondre en un même substrat la réalité de la propagande " aubertiste » et une quelconque opinion réunionnaise dont on suppose qu'elle ne serait que le reflet de sentiments plus profonds. La réaction des subalternes fait cruellement défaut dans ce domaine.

Soucieuse de contrôler les possibles oppositions, l'administration coloniale établit alors un contrôle presque systématique sur le service postal dès la signature de l'armistice. Le moral de la population fait l'objet d'une attention particulière que les services de la propagande s'empressent d'analyser afin de peaufiner leur rhétorique. Tant que la censure n'est pas perçue comme telle au sein de la société, la liberté d'expression est manifeste. Cette situation n'a cependant de réalité que jusqu'au mois d'août 1940, période à partir de laquelle le nombre de courriers tend à diminuer tout autant que la sincérité des écrits. L'opinion est jugée très instable, "désemparée " ${ }^{35}$, et "le chaos moral s'est accentué » ${ }^{36}$. Le désarroi moral, "né de l'ignorance du passé et de l'incertitude de l'avenir, paraît avoir anesthésié les gens » ${ }^{37}$. Malgré cette "torpeur coloniale ", trois tendances principales sont mises en évidence par les services de la censure : "La première milite en faveur du Gouvernement du Maréchal Pétain et pour la continuation de la guerre par les colonies. La deuxième se rallie autour du général de Gaulle et la troisième est pour l'alliance franco-anglaise. La majorité de la population, en raison de ses attaches familiales avec les Mauriciens [...], semble appuyer cette dernière tendance. $»^{38}$

Les bravades belliqueuses restent nombreuses dans les premiers courriers ouverts, et le soutien à l'Angleterre est une constante dans les premiers mois du régime " aubertiste ». Les Mauriciens de l'île témoignent ainsi beaucoup de sympathie pour la France, mais, chose très blessante pour l'amour-propre national, la considèrent comme ayant besoin d'un tuteur. Leur ardeur est cependant vite calmée et rapidement ils « évitent de parler des relations de la France avec son ex-alliée ${ }^{39}$. Les liens familiaux et les intérêts économiques entrent, pour une large part, dans cette idée de rapprochement. Cet état d'esprit est confirmé par $M^{\text {gr }}$ Cléret de Langavant dans son journal : "Ici à la Réunion, il y a cependant un fort parti d'opposition qui regrette

35. ADR 1M604, Rapport contrôle postal, juin 1940.

36. Ibid.

37. Rapport contrôle postal, juin 1940, op. cit.

38. Ibid.

39. Ibid. 
qu'on ne soit pas livré à l'Angleterre. Ce que l'on voit surtout c'est que la France ne pourra sans doute pas nous acheter nos sucres ni assurer notre ravitaillement. L'Angleterre ayant la suprématie navale pourrait nous ravitailler et en même temps prendre notre sucre. $»^{40}$

Il est vrai que le clergé réunionnais soutient le gouverneur dans sa politique de fidélité au Maréchal, l'Évêque n'hésitant pas à parler d'unanimité. Dans le même temps, le soutien aux gaullistes est-il une réalité durant ces premiers mois de l'installation du nouveau régime? Plus largement concerné par les élites, ce soutien suppose une connaissance plus précise des rares informations émanant de médias proposant une alternative. Il n'est donc pas exceptionnel de rencontrer certaines agitations au sein même des administrateurs en place ou par le biais des notables de chaque commune. Certains poussent leur engagement jusqu'à dénoncer la politique menée par le gouverneur Aubert en écrivant directement à ses services. Tel est le cas de cet ancien fonctionnaire, Roger Guichard, condamné à trois mois d'emprisonnement en octobre 1940 pour ses propos antigouvernementaux. Ce dernier avait envoyé une lettre dans laquelle il dénonçait l'autoritarisme factice de Pierre Aubert, car ce dernier ne pouvait « obtenir que l'obéissance du forçat à sa chaîne " ${ }^{41}$. Il proférait ensuite des menaces à peine voilées en lui rappelant que "nous sommes plus forts que vous, car ici nous sommes les Maîtres ${ }^{42}$. Ces velléités belliqueuses et le soutien aux Forces françaises libres ne cessent d'inquiéter les services de la censure qui rappellent que "l'action du général de Gaulle est unanimement approuvée, on la considère comme la seule porte de sortie de la situation actuelle $»^{43}$. Certains courriers entrevoient même une collusion entre Pétain et de Gaulle afin de sauver la France. Cette adhésion de principe ne signifie aucunement une quelconque structuration d'un mouvement gaulliste sur l'île. Il faut attendre l'année 1941 pour que ce mouvement recrute des partisans et organise des actions clandestines.

La diffusion de plus en plus massive des informations reçues de Vichy sur la vie de l'Empire contribue nettement à renverser l'opinion. Les communications radiodiffusées par le poste Radio Saint-Denis sont dans le même but largement utilisées. D'autre part, l'attaque de la flotte française à Mers el-Kébir et la tentative de débarquement à Dakar réactivent les sentiments anglophobes d'une frange de l'opinion réunionnaise. La lecture des lettres confirme l'évolution des esprits, très favorables à la personne du Maréchal, "avec qui chaque jour davantage se confond la patrie ${ }^{44}$. Désormais, les prises de position en faveur de Pétain deviennent légion.

La confiance exprimée envers le Maréchal ne s'inscrit que rarement dans les idéaux de la Révolution nationale ou dans le respect des principes

\footnotetext{
40. Journal de $\mathrm{M}^{\mathrm{gr}}$ Cléret de Langavant, Archives diocésaines de La Réunion (non côté).

41. Rapport contrôle postal, juin 1940, op. cit.

42. Ibid.

43. Ibid.

44. ADR 1M605, Rapport contrôle postal, février 1941.
} 
de la collaboration. C'est le sauveur de la France qui est mis à l'honneur. Le sort des Réunionnais est entre les mains de "l'honorable vieillard " qui est à même de faire comprendre " combien l'Angleterre a fait plus de mal à la France que son ennemi l'Allemagne $»^{45}$. Rares sont les échos d'une réelle communion de pensée avec l'occupant que les autorités se font un devoir d'étouffer avec célérité et sévérité. Tel est le cas du bravache Arsène Roufli en juillet 1940 qui n'hésite pas à exhiber une croix gammée. Cette manifestation isolée et la réaction subséquente des autorités illustrent l'attachement de l'administration "aubertiste " à un maréchalisme de circonstance plutôt qu'à un pétainisme d'ordre idéologique.

In fine, même si le loyalisme peut apparaître comme une forme de fanatisme ${ }^{46}$, propre à étayer la thèse d'un " décervelage $~^{47}$ de la part de l'Administration coloniale ; d'autres principes ont certainement guidé le ralliement à Vichy. Selon les hypothèses énoncées par Henry Rousso, des facteurs convergents entre la métropole et les colonies peuvent être mis en parallèle. Certes la popularité de Pétain ne fait aucun doute et débouche sur une dévotion d'autant mieux imprégnée qu'elle s'inscrit dans un cadre patriotique exacerbé autour du héros de Verdun. Le soutien de la hiérarchie catholique a certainement été favorable également à l'acceptation du nouveau régime. $\mathrm{M}^{\mathrm{gr}}$ Cléret de Langavant a pu trouver dans les principes traditionalistes défendus par le nouveau régime une certaine communion de pensée. Pourtant, la crainte d'une ingérence du gouverneur dans les affaires du diocèse et un jugement peu élogieux sur sa personne dans son journal laissent penser à un jeu de dupe plutôt qu'à une véritable communion sur les principes.

Le soutien de la plus grande partie de l'Administration est de fait acquis dès la signature de l'armistice. On ne peut cependant y voir une adhésion idéologique mais plus simplement une passivité à l'origine selon Pierre Aubert d'une somnolence administrative. Même s'il reconnaît que "les fonctionnaires coloniaux et municipaux font généralement preuve d'une louable activité. Il est cependant à regretter que la majorité soit installée dans une vie quiète et casanière, $[\ldots]$ une vie amollissante de retraités provinciaux $»^{48}$. De même, l'inertie des populations, engluées dans les difficultés du ravitaillement, reste une constante au cours de cette période. Le manque d'informations et la maîtrise difficile des véritables enjeux d'une telle affiliation devaient certainement échapper au plus grand nombre. Certes, "on trouve les traces d'une sincère compassion et de compréhension à l'encontre des Français de la métropole " ${ }^{49}$, mais "la guerre apparaît lointaine $\|^{50}$. C'est ce que constatent les services de la censure en soulignant que la population ne commente pas les mesures prises par le gouvernorat.

45. ADR 1M605, Rapport contrôle postal, octobre 1941

46. Jennings Eric, op. cit.

47. ANOM 1 Affpol 1133, René Pleven à Henri Hoppenot, 30 juillet 1943.

48. Rapport politique Pierre Aubert (1941), op. cit.

49. ADR 1M605, Rapport du contrôle postal, juillet 1941.

50. ADR 1M605, Rapport du contrôle postal, décembre 1940. 
La population se contente d'obéir, "la majorité de bon cœur et la minorité de beaucoup plus faible subissent les événements avec une ferveur anémiée ${ }^{51}$. Les rares propos outrageants à l'égard du chef de l'État et des autorités locales sont surtout liés aux difficiles conditions économiques. Ils ne dépassent guère la sphère parfois houleuse des boutiques et des bars mais constituent une menace jugée suffisamment sérieuse pour aboutir à des condamnations. De même, l'absence d'alternative politique crédible a certainement été favorable à l'installation du régime "aubertiste ». L'allégeance au positionnement politique de Madagascar illustre cette argumentation avec d'autant plus d'acuité que les forces militaires à la disposition de Pierre Aubert ne permettaient pas d'assurer de façon autonome la défense de l'île. De la même manière, l'espoir de revaloriser l'action de l'État après une période considérée comme instable a pu influer sur l'adhésion de certains. Après les tensions issues de la période du Front populaire, certains notables ont pu voir en Vichy une opportunité pour contrecarrer les projets du camp progressiste réunionnais et régler leurs comptes avec une République jugée trop instable et peut-être trop sociale : «La République est morte. Un bel enterrement et n’y pensons plus... la France renaîtra. $»^{52}$

Enfin, nous pouvons émettre l'hypothèse d'une filiation par une volonté accrue de reconnaissance de la part de la métropole. Le mythe colonial de Vichy est ainsi à l'origine d'une rhétorique où « le maintien de la France éternelle et l'illusion de la puissance malgré la défaite $»^{53}$ ne pouvaient provenir que de l'Empire. En mettant en exergue la fierté créole et le poids politique de l'Empire, La Réunion a pu «trouver dans le malheur de la patrie l'occasion magnifique de resserrer autour d'elle, d'affirmer entre elles et avec la métropole un renouveau de solidarité impériale " ${ }^{54}$. Cette union retrouvée avec la mère patrie permet selon cette logique d'atténuer « le régionalisme réunionnais, par trop accusé dans les temps passés et consistant à raisonner uniquement en créole " pour céder « le pas à l'idée de la France d'abord $»^{55}$.

\section{Les cérémonies exaltant l'Empire}

Toute la force d'un discours de propagande se résume dans sa mise en scène. Les services coloniaux en comprennent d'autant mieux les enjeux qu'ils se doivent de raffermir la confiance envers un régime en proie aux doutes et aux contestations. Initiées par le ministère des Colonies, les célébrations d'une "Semaine impériale » en 1941 puis d'une «Quinzaine impériale » l'année suivante permettent aux différents territoires de l'Empire

51. ADR 1M605, Rapport du contrôle postal, mars 1941.

52. ADR 1M605, Rapport R.G., août 1940.

53. Pascal Blanchard et Ruth Ginio, op. cit.

54. ADR 1M4003, Allocution Pierre Aubert, 19 novembre 1941.

55. Rapport du contrôle postal, juillet 1941, op. cit. 
d'apporter leur contribution au principe de la régénération nationale. La célébration des colonies et la reconnaissance de leurs forces vives impulsent une nouvelle dynamique dont le moteur alimente l'idée d'un relèvement grâce à l'Empire. Ce dernier devient ainsi un "mythe consolateur " ${ }^{56}$ assurant le maintien d'une France éternelle malgré les désillusions engendrées par la défaite.

De toutes les cérémonies et festivités organisées par le gouvernorat de Pierre Aubert, la "Semaine impériale » du 15 au 21 juillet 1941 est sans nul doute la plus aboutie. Elle doit amorcer dans l'esprit du gouverneur l'aube d'un relèvement ${ }^{57}$. Cette projection vers l'avenir n'a pour but que de rappeler la communion de pensée et de destin unissant la colonie avec sa métropole. Les discours insistent ainsi sur les enjeux historiques et politiques d'une telle reconnaissance des valeurs et des vertus de l'Empire. Rappelant la fidélité ancestrale à la patrie et les mérites de son nouveau guide, les logiques discursives s'inscrivent également dans une valorisation des caractères spécifiques de la colonie. Leurs auteurs sont issus des plus hautes instances coloniales et sont censés représenter une caution morale sans faille. Que ce soit $\mathrm{M}^{\mathrm{gr}}$ Cléret de Langavant, Raoul Nativel, Hyppolyte Foucque, Raymond Vergès ou certains enseignants du lycée de Saint-Denis, ces derniers développent une rhétorique d'exaltation des forces réunionnaises.

La tonalité est donnée par le gouverneur lors de l'inauguration des festivités. Il rappelle que trois siècles d'histoire ont dicté à La Réunion, " fille lointaine mais aimante ${ }^{58}$, son devoir envers la mère patrie. Malgré la souffrance de la défaite et les difficultés rencontrées au cours de cette " année cruelle ", les liens ne se sont jamais distendus. L'île Bourbon "n'a jamais douté de son destin » et envoie son "affectueux salut à la France immortelle $"^{59}$. La fidélité de destin et la ferveur patriotique constituent le socle d'une argumentation moralisatrice. Les festivités s'articulent autour de la radiodiffusion des discours les plus marquants, de l'organisation de cérémonies commémoratives, de la collecte de fonds et de l'inauguration sur le terre-plein du Barachois de l'esplanade du maréchal-Pétain suivie de la prestation de serment des membres de la Légion française des combattants.

Les discours radiodiffusés ont une portée pédagogique et relatent les grandes figures d'une histoire coloniale édifiante portant à la vertu par l'exemple. Les arguments se sont accordés pour ressusciter au micro les pionniers de l'œuvre civilisatrice de la France. Les panégyriques énoncés sur Ferry, Montcalm, Dupleix, Bugeaud, Savorgnan de Brazza ou Marchand ne font que refléter le poids de la culture impériale dans

56. Pascal Blanchard et Ruth Ginio, op. cit, p. 370.

57. "La Réunion vous parle. Semaine de la France d'outre-mer à l'île de La Réunion (15 au 15 juillet 1941) ", Saint-Denis, 1941.

58. Ibid.

59. Ibid. 
l'imaginaire collectif. Cette logique est partagée par l'ensemble des notables conviés aux festivités. C'est ainsi que Raymond Vergès est invité à s'exprimer sur l'œuvre coloniale de la France. Il évoque, en qualité de chef de la santé, les bienfaits de la colonisation dans le domaine médical et rappelle incessamment les "vertus de notre race et son rôle dans le monde $"^{60}$. Les Réunionnais ont ainsi pu s'enorgueillir d'une "histoire glorieuse ${ }^{61}$ démontrant la solidité des liens qui l'unissent à la mère patrie. Les propos tenus par les chroniqueurs insistent sur les attentes impériales du nouveau régime. Si les Réunionnais doivent rester «fiers et forts dans l'épreuve ", ils doivent également mettre en avant leurs qualités pour donner l'exemple à la patrie meurtrie. Certes l'âme créole «conserve en même temps que ces caractères ancestraux sa tendance à discuter de tout. Mais sa contexture étant strictement à l'image de l'âme même de la France, l'heure est particulièrement favorable à l'affirmation d'une identité que je dirai substantielle et congénitale $»^{62}$. L'affirmation d'une identité culturelle à la fois commune et différente est au cœur du concept de la « Plus Grande France », une nouvelle France impériale gardienne des valeurs de la nation. Plus qu'une articulation d'identités multiples, les discours mettent surtout en avant une communauté identitaire. Cette nouvelle France impériale est donc au cœur de la constitution et de la redéfinition d'un nouveau principe national où les devoirs n'en deviennent que plus nobles.

Comme le rappelle le secrétaire général, cet "amour fervent de La Réunion pour la France ${ }^{63}$ trouve ainsi son expression concrète dans la contribution des populations aux œuvres du Secours national. C'est, selon son argumentation, le "plus réconfortant témoignage de solidarité de la population réunionnaise, un gage plus sûr des liens indéfectibles qui l'unissent à la grande patrie " ${ }^{64}$. Cette générosité trouve son origine dans la grandeur d'âme créole et dans son ardent désir de témoigner son esprit patriotique. Elle va à l'encontre de ceux qui «doutent encore du destin français » et essayent de faire basculer l'île dans le camp de la France Libre, de l'Angleterre, "des Juifs, des francs-maçons et des communistes [qui achèvent] rapidement la désagrégation totale de la nation française ${ }^{65}$.

Afin de guider l'action des Réunionnais dans cette œuvre de régénérescence, "d'unir tous les Français pour ne faire qu'un bloc ", le soutien sans concession au gouvernement de Vichy semble de mise. Selon l'évêque du diocèse, cette impérieuse nécessité est guidée par la volonté divine et dans le credo des trois vertus théologales que sont la Foi, l'Espérance et la Charité. "La foi en la France et en son chef. Ce sera de croire en elle, en sa puissance de vie nouvelle, en sa résurrection comme nation libre, indépendante de toute autre. L'espérance est basée sur la foi, elle en est

60. Ibid.

61. Discours Raoul Nativel, «La Réunion vous parle... ", op. cit.

62. Ibid.

63. Avant-propos de Jean Rivière, "La Réunion vous parle... ", op. cit.

64. Ibid.

65. Sermon de $\mathrm{M}^{\mathrm{gr}}$ l'évêque de Saint-Denis, 13 juillet, "La Réunion vous parle... ", op. cit. 
la conséquence immédiate. Si nous avons foi, si nous croyons à la France, nous attendrons avec confiance son relèvement. Charité enfin, car seule la charité est capable de faire grand et durable. $»^{66}$

Le redressement de la patrie sera donc la résultante des efforts de chacun et de l'union des forces vives de la nation. Fort du succès de cette manifestation, le gouvernorat décide de renouveler l'expérience l'année suivante du 15 au 31 mai 1942. Les enjeux sont d'autant plus grands que La Réunion fête alors le tricentenaire de sa destinée française. Les festivités nécessitent donc un éclat digne de la commémoration. Un comité est ainsi constitué dans le but d'organiser des manifestations littéraires et des reconstitutions historiques. Le tout est relayé par un comité de propagande dont les trois directeurs des journaux Chantecler, Le Peuple et Le Progrès sont invités à participer sous le contrôle du chef de cabinet. Afin de vivifier les manifestations, un comité de participation se structure également autour de la Légion française des combattants et des volontaires de la Révolution nationale.

La propagande établit rapidement son plan d'attaque en diffusant sur les ondes des slogans mobilisateurs, des chroniques historiques à caractère régional et des œuvres poétiques célébrant l'Empire. Afin de donner une caution scientifique à cette remémoration d'une identité nationale partagée, les organisateurs invitent des historiens de renom comme Albert Lougnon pour entériner le principe de l'implantation initiale d'une souche française sur l'île. Cette matrice nationale commune explique que les Créoles ont conservé «leur âme française que l'éloignement n'a jamais séparée de la grande patrie $"{ }^{67}$. Les premiers descendants de Bourbon ont apporté avec eux « les caractères distinctifs de notre race " qu'ils ont su conserver intacts. Cette préservation des attributs historiques de la nation explique que "sur la souche vivace et forte des premiers colons, le rameau créole pousse dru, vigoureux, solide, nourri de la sève marine et paysanne du vieux pays $\|^{68}$. On y retrouve, "avec des archaïsmes touchants et les transpositions de la vie des tropiques, le "climat" provincial d'une France toujours proche et lointaine $"^{69}$. Ce retour sur le passé glorieux de l'île s'inscrit dans un régime d'historicité spécifique propre aux acteurs de cette période. Las d'attendre les avancées politiques et sociales inhérentes au processus d'assimilation, une frange de la population a pu se laisser bercer par une certaine nostalgie mémorielle. Ces sentiments trouvent notamment leurs origines dans la valorisation patrimoniale initiée depuis la fin de la Grande Guerre. Plus instantanément, le contexte stratégique de l'année 1942 explique le redoublement des efforts afin de mobiliser l'opinion. En effet, depuis le mois de mai, les Britanniques entreprennent des opérations militaires sur

66. Ibid.

67. ADR 1M4045, Discours de Vincent Boyer de la Giroday.

68. ADR 1M4045, Allocution Pierre Aubert à l'occasion de l'ouverture de la Quinzaine impériale.

69. Ibid. 
Madagascar. La sécurité de l'île est désormais menacée, et « cette quinzaine impériale s'ouvre hélas dans le deuil des héroïques défenseurs de Diégo, dans l'angoisse des invasions possibles ${ }^{70}$. Le tricentenaire se transforme alors en un cri d'amour pour la France qui s'incarne dans le rappel de "tout un passé de gloire et d'héroïsme $»^{71}$. La menace britannique permet la remémoration "de toutes les guerres où les nôtres sont tombés sous les balles anglaises ". Le souvenir de 1810 où "ces pères hardis prirent leurs fusils » incarne cette volonté de «se grouper et mourir plutôt que d'être anglais $»^{72}$.

Au-delà des idées propagées par les autorités coloniales qui correspondent à une adhésion de principe sur le sens du devoir par le prisme colonial, il convient de s'interroger sur les modalités d'application d'une régénération impériale et sur les moyens déployés par les services de la propagande.

\section{LA RÉGÉNÉRATION NATIONALE PAR L'EMPIRE}

Propagande...

Les objectifs explicites de la propagande se comprennent selon une double acceptation chronologique. Il s'agit à la fois de donner une perspective aux Réunionnais pour un avenir considéré comme incertain mais également de faire le procès du passé afin de s'inscrire dans une optique régénératrice.

Initiée et organisée depuis la métropole grâce au haut-commissariat à la Propagande puis au secrétariat général à l'Information, elle s'est de suite appliquée à La Réunion. Cependant, l'éloignement de l'île par rapport au pouvoir central laisse supposer que l'éclat accordé aux cérémonies et la force des discours ne pouvaient dépendre que de la volonté des responsables coloniaux alors en poste. Le poids des mots dépend effectivement de celui qui les énonce et de leur mise en scène. Dans ce domaine, le rôle joué par Jean-Jacques Pillet a été primordial puisqu'il disposait des leviers nécessaires à leur organisation et leur contrôle. Les aléas de sa carrière expliquent en partie le zèle déployé à son poste de chef de cabinet. Promu en décembre 1938 sous l'égide du gouverneur Joseph Court, il s'inscrit pleinement dans les réformes engagées et prend peu à peu de l'assurance dans ces fonctions. Il supplée régulièrement Pierre Aubert lorsque ce dernier est absent, élargissant ainsi ses fonctions aux différentes instances de la censure telles que le bureau de la Presse-Information et Propagande. Par ce biais, il contrôle la publication de nombreuses brochures et participe à la

70. ADR 1M4045, Chantecler, 18 mai 1942.

71. Ibid.

72. Ibid. 
rédaction de certains articles dans le journal Chanteclerc. Souhaitant former un corps de propagandistes dévoués, il élargit ensuite ses compétences à la Garde du Maréchal et au Comité de propagande Pétain. Comprenant que le problème n'est pas tant d'être convaincu que d'user des armes de la persuasion à des fins politiques, il a su propager des fantasmes divers dont la répétition des termes a pu ancrer dans les esprits une subjectivité inconsciente. C'est en ce sens que la propagande repose sur un état d'esprit apte à la recevoir mais aussi à la propager. Considérée comme une "arme de choix $»^{73}$, elle s'appuie sur le moral de la population qu'elle essaye tout à la fois de sauvegarder et de manipuler. Elle ne peut donc être efficace que dans le cadre d'une popularité supposée du nouveau régime.

L'environnement réunionnais constitue à cet effet un terreau certainement propice. En effet, pour être crédible et persuasive, la propagande se doit de proposer "un dialogue politique ordonné autour d'un ensemble de conventions ou de préjugés $\${ }^{74}$ capables d'influencer et de manipuler. Or, le contexte met en lumière l'adéquation entre certaines valeurs de la Révolution nationale et les principes organisationnels de la société coloniale. L'importance accordée à la famille dans le cadre d'une démographie galopante laisse entrevoir une acceptation du principe de hiérarchisation naturelle d'une société plus proche de la terre où la difficile application des valeurs démocratiques atténue le principe de l'individualisme. Il est ainsi plus facile d'enseigner la devise Travail, Famille, Patrie que Liberté, Égalité, Fraternité en milieu colonial ${ }^{75}$. Autant de conventions et de préjugés partagés avec les idéologues de Vichy et leurs partisans coloniaux.

Les moyens de communication utilisés par les services de la propagande témoignent d'un contrôle total du champ médiatique. Si les expositions et les conférences ont pour but de convaincre les élites, la force persuasive du cinéma, de la radiophonie et de la presse concerne un public plus large. Cette dernière, fortement contrôlée, s'appuie sur des publications parfois anciennes et idéologiquement contrastées. Certes, Le Peuple et Le Progrès ne montrent que très rarement de la sympathie pour la Révolution nationale et le régime de Vichy mais participent à la fondation d'un imaginaire collectif en relayant les informations officielles. L'absence de positionnement politique devait sans doute laisser le lecteur dubitatif quant au bien-fondé des informations présentées. Tel n'est pas le cas de $\mathrm{La}$ Démocratie de Raphaël Babet qui encense le régime en place, du journal Servir de René Payet qui sert de relais aux idéaux de l'Action française, de La Vie catholique et du bihebdomadaire Dieu et Patrie qui ne sont que les porte-parole de la ligne politique traditionaliste suivie par $\mathrm{M}^{\mathrm{gr}}$ Cléret de Langavant. Accentuant leurs efforts de propagande, les autorités coloniales décident également de lancer dès novembre 1940 le journal Chantecler. Cet

73. Jean-Pierre Azema et Olivier Wieviorka, Vichy, 1940-1944, Paris, Perrin, 1997, p. 110.

74. Ruth Ginio, "La propagande impériale de Vichy ", in Pascal Blanchard, Sandrine Lemaire et Nicolas Bancel (éds.), op. cit, p. 118.

75. Ibid. 
hebdomadaire du pouvoir "aubertiste » est certes administré par l'ingénieur Eugène Poisson mais dépend in fine de la ligne éditoriale définie par Jean-Jacques Pillet et de quelques rédacteurs influents comme le conseiller à la cour d'appel Eugène Thébault ou le secrétaire général Rivière.

Prenant conscience de la force communicative des nouveaux médias, les autorités tentent dans le même temps d'initier une dynamique cinématographique. Les propagandistes croient au pouvoir des images et diffusent des films vantant tout à la fois les mérites de l'Empire et de la Révolution nationale. Provenant des services de la propagande de Madagascar, ils mettent surtout en scène les bains de foule du Maréchal, les principes d'une régénération nationale (La France en marche) ou les événements tragiques concernant l'Empire (Mers el-Kébir, Dakar). Ces films sont projetés dans les différentes communes de l'île et mobilisent de nombreux spectateurs. Ces projections sont l'occasion d'une mise en scène spécifique où les édiles locaux se doivent d'être présents en signe d'allégeance.

La censure est mise en place progressivement tout en s'appuyant sur les mesures prises lors de l'entrée en guerre. Ces dernières disposent que "désormais, tous les imprimés, dessins, ou écrits de toute nature, ainsi que les émissions radiophoniques et les projections cinématographiques, non soumis au contrôle préventif du service général des informations sont interdits $»^{76}$. Les modalités d'application deviennent de plus en plus draconiennes avant de s'inscrire dans une logique proprement vichyssoise à partir de 1941. La création du bureau Presse-Information et Propagande, puis la promulgation le 22 septembre d'un nouvel arrêté disposant que "la publication des journaux, ouvrages, imprimés, dessins ou écrits de toute nature est subordonnée au visa préalable du chef de bureau $»^{77}$. Afin de prévenir la divulgation des « informations militaires de toute nature, de toute nouvelle, information, écrit, dessin, imprimé susceptible d'avoir une influence fâcheuse sur l'esprit de l'armée et de la population $»^{78}$, le contrôle de la presse devient ainsi total.

D'autre part, le contrôle des diffusions radiophoniques est renforcé aux vues de l'impact qu'elles génèrent sur le public. Seules sont autorisées les émissions produites par Radio Saint-Denis étroitement contrôlées par Jean-Jacques Pillet. De même, ce dernier se prémunit de toutes diffusions d'ordre privé « que le haut-parleur ou diffuseur du poste soit situé ou non sur la voie publique $»^{79}$. Rares sont les municipalités habilitées à diffuser sur haut-parleur les animations musicales et les bulletins de presse de la radio dionysienne. N'ayant qu'une diffusion limitée à deux créneaux quotidiens, les autorités craignent une écoute des émissions de la France Libre (Radio Brazzaville) et de leurs alliés anglais (Radio Maurice). C'est pourquoi, le 3 octobre 1941, un autre arrêté limite encore plus les possibilités d'écoute

76. ADR série 8K, JOR, Arrêté du 18/09/1939.

77. Ibid.

78. Ibid.

79. Ibid. 
en prohibant la réception ou l'audition des postes britanniques ou étrangers qui se livrent à de la propagande antinationale. Les thèmes propagés par Radio Saint-Denis présentent de nombreux traits communs avec ceux développés par la propagande métropolitaine. Idéologie de combat, les thèmes les plus récurrents concernent les ennemis du nouveau régime ${ }^{80}$. À l'anglophobie traditionnelle qui permet d'exalter l'Empire français, s'ajoutent de violentes attaques contre les partisans de la France Libre. Au fur et à mesure de l'évolution des rapports de force et des alliances consécutives, la liste des ennemis potentiels s'élargit aux Soviétiques et aux Américains. Dans l'esprit des auteurs, il s'agit principalement de lutter contre les internationales que constituent à leurs yeux le capitalisme et le communisme. Cependant, l'adhésion aux idéaux de la Révolution nationale et l'admiration sans voile à la personne du maréchal Pétain constituent le ferment à la base de l'unité. En ce sens, la propagande sur l'île rejoint sur de nombreux aspects celle initiée par les lobbies coloniaux de Vichy. La singularité du combat des Réunionnais est cependant affirmée en filigrane tout au long des chroniques radiodiffusées.

Les injonctions morales associées aux valeurs défendues par les élites réunionnaises constituent ainsi un formidable miroir des mentalités coloniales. En ce sens, la propagande est plutôt conformiste et repose sur des présuppositions sociologiques où le caractère émotionnel est déterminant. Plus qu'une propagande d'agitation reposant sur la différenciation puis la condamnation des ennemis définis par le régime, on décèle plutôt une volonté de conformation aux idéaux d'une Révolution nationale que l'on voudrait initiée par l'Empire.

\section{... et embrigadement}

Cette condition politique étant établie, les autorités se sont empressées de créer des relais pour contrôler et influencer l'opinion. Dans leur esprit, l'endoctrinement des masses supposait de dépasser le simple cadre du discours pour s'inscrire dans une logique participative et d'encadrement de la société. Le recrutement de nombreux volontaires au sein de la Légion française des combattants, de la Garde du Maréchal ou du Comité de propagande Pétain démontre tout à la fois la force coercitive du régime et l'adhésion idéologique d'une frange de la population.

De prime abord, la transplantation à La Réunion d'une Légion française des combattants répond à une obligation légale puisque toutes les dispositions juridiques établies pour la métropole s'appliquent en principe au reste de l'Empire. La Légion eut le rôle de vecteur et de garant de la Révolution nationale en qualité de corps emblématique des forces vives de la nation. Elle n'est réellement structurée qu'en juillet 1941 avec la

80. ANOM BIB AOM $/ / 43794$, Entre nous... Bulletin de propagande du poste de radio de Saint-Denis 19401942, Éditions du bureau de la Presse-Information-Propagande Imp. Casal, 1942. 
publication d'un organigramme spécifique. Les responsables sont principalement des propriétaires terriens (33\%), des employés de mairie $(18 \%)$ et des employés du secteur privé (10\%). Certains membres du clergé $(8 \%)$ y sont représentés ainsi que des pensionnés et des retraités (10\%). La Légion bénéficie également du soutien de l'Association des anciens combattants de l'île qui décide de s'y associer lors des cérémonies du 11 novembre 1940.

Les discours clament avec force l'héritage de la Grande Guerre et mettent en avant l'esprit patriotique des anciens combattants. Les légionnaires s'inclinent ainsi avec "ferveur et avec respect devant les camarades qui sont tombés pour la défense de la patrie et l'honneur du drapeau $»^{81}$. Il s'agit de rester fidèle à la mémoire de ceux qui sont tombés au champ d'honneur, " c'est-à-dire à rester fidèles au drapeau dans les plis duquel ils ont été ensevelis " ${ }^{82}$. Désormais, "les rescapés de Verdun, égarés pendant vingt ans par de mauvais bergers, font solennellement le serment d'être derrière le Maréchal pour refaire une France grande, forte et digne de son passé ${ }^{83}$. Les légionnaires se sentent investis d'une mission "indispensable au salut et à la rénovation de la France ${ }^{84}$. C'est un devoir civique que l'on compare à un "ordre de mobilisation".

C'est par un arrêté du gouverneur du 29 novembre 1941 que la Garde du Maréchal est instituée sous la responsabilité de Jean-Jacques Pillet. La formation de cette structure aurait été initiée par la venue du lieutenant Gresset sur l'île en octobre 1941. Ce dernier est un propagandiste de Vichy en poste à Madagascar qui fut directement diligenté par Gabriel Jeantet alors inspecteur de la Propagande. Ce faisceau d'activistes ne compte " en son sein que des membres sûrs de leur doctrine nationale ${ }^{85}$, dont les chefs de groupe sont issus pour une bonne part du scoutisme, du Lycée et des jeunesses des écoles chrétiennes. Leur mission est de «faire connaître et comprendre à la population et plus spécialement dans les milieux de jeunes l'œuvre du Maréchal ; faire aimer sa personne ; répandre les disciplines de la Révolution nationale ${ }^{86}$. À terme, l'objectif est de convaincre des sympathisants et de les enrôler au sein de la Légion française des combattants et des volontaires de la Révolution nationale. Les membres s'engagent à agir uniquement en Français dans une «France française, une France propre et forte, une France chrétienne ${ }^{87}$. Ils doivent veiller à lutter contre les ennemis de la Révolution nationale que l'on encourage à dénoncer auprès des autorités. Les professions de foi exprimées dans les lettres de candidature dénotent un engagement idéologique sans faille et dressent un profil sociologique précis des adeptes de la Garde.

81. ADR 1M4044, Allocution Roger Payet, $1^{\text {er }}$ novembre 1941.

82. Allocution Rieul-Dupuis, "La Réunion vous parle... ", op. cit.

83. Ibid.

84. ADR 1M4045, Allocution Hyppolite Foucque, 31 mai 1941.

85. ADR 1M4023, Directives de la Garde du Maréchal, 1942.

86. Ibid.

87. ADR 1M4023, Serment pour la Garde du Maréchal. 
Cet esprit transcende les classes d'âge afin de mobiliser les plus jeunes membres qui regrettent de n'avoir pu s'engager dans le conflit et défendre le drapeau. Cette connotation guerrière vivifie un patriotisme belliqueux pourtant peu en phase avec la réalité impériale. L'analyse du profil des 63 membres enrôlés sur le chef-lieu dionysien montre que l'âge moyen est de 22 ans avec un écart-type peu conséquent. Dans ce projet de régénération où doit émerger un homme nouveau, la jeunesse occupe ainsi une place essentielle ${ }^{88}$. La grande majorité des membres n'a donc pas connu la Grande Guerre mais a été bercée par son souvenir et son lot de commémorations. Le plus souvent diplômés, ils appartiennent surtout à la classe moyenne et travaillent pour un bon nombre d'entre eux dans les services de la colonie. Le profil des parents révèle une forte proportion de fonctionnaires et notamment d'enseignants. Dans les autres communes de l'île, les membres sont légèrement plus jeunes, moins diplômés et de condition plus modeste. Fort de leur réseau de près de 400 membres, parmi lesquels sont inscrits les fils des notables les plus éminents, l'action de la Garde repose sur le travail de propagande entrepris par le Comité de propagande Pétain sous les ordres du maire de Saint-Denis, Armand Barau. Ce Comité « est la pointe d'avant-garde du vaste mouvement de Révolution nationale dont la Légion française forme l'assise puissante $»^{89}$. L'arrêté définissant ses fonctions lui assure la responsabilité des thèmes développés par la propagande en partenariat avec Jean-Jacques Pillet. Cependant, en complément de l'action menée par ce dernier, la vocation du Comité est d'abord de diffuser les idéaux de la Révolution nationale par un travail incessant en partant de la base grâce à l'infiltration de propagandistes bénévoles.

In fine, la propagande d'agitation peu décisive et celle plus active d'intégration ont donc combiné leurs efforts pour renforcer la légitimité du nouveau régime et bercer l'opinion dans l'illusion d'une régénération impériale. Profitant de la relative passivité des masses, les services du gouverneur ont pu croire à un réel attachement de l'île aux idéaux ainsi défendus. Pourtant, qui ne dit mot ne consent pas forcément si l'on considère que le mutisme et le conformisme apparents sont des constantes de la condition politique coloniale. De même, la participation active de nombreux Réunionnais aux festivités publiques ou leur embrigadement au sein des différentes associations provichystes ne signifient nullement une approbation sur les principes. Tout au plus s'agit-il d'un jeu de dupes ou d'un accommodement contraint pour une grande partie de la population en proie aux doutes et aux difficultés matérielles. On ne peut cependant occulter le zèle d'une minorité dont l'opportunisme devait se conjuguer à une réelle adhésion idéologique.

88. Jacques Cantier, "Un enjeu essentiel. Vichy et les jeunes dans l'Empire français ", in Jacques Cantier et Eric T. Jennings, op. cit., p. 91-115.

89. ADR 1M4023, Discours Armand Barau, 26 mars 1942. 


\section{CONCLUSION :}

La rhétorique de la Révolution nationale s'inscrit de manière congruente dans une volonté de réforme impériale et de reconnaissance accrue des valeurs coloniales. L'adhésion d'une partie de la population aux idéaux d'une nouvelle France régénérée par son Empire repose sur un patriotisme traditionnel se nourrissant du " pays réel » et des éléments constitutifs de la société. On peut y voir le rejet d'un patriotisme révolutionnaire dont la finalité assimilatrice tarde à montrer les premiers signes tangibles. La vérité n'est donc pas dans les faits, qui n'en sont que la partie visible. Elle est dans la compréhension des raisons qui l'ont fait accepter par une partie de l'opinion réunionnaise. Le soutien à Vichy s'explique ainsi par les déceptions générées par le système républicain. De manière concomitante, les idéaux défendus par les administrateurs coloniaux sur l'île ont pu trouver des points de convergence sur cette critique de la politique assimilatrice. En effet, ces derniers ont accompli l'essentiel de leur carrière dans les territoires de l'Empire où le principe d'assimilation n'était qu'une chimère. Ils ont donc manifesté leurs frustrations par une adhésion pleine et entière à l'idéologie vichyssoise. Pourtant, l'intérêt de la colonie était de préserver les acquis républicains qui faisaient de La Réunion un territoire relativement privilégié au sein du monde colonial français ${ }^{90}$. Cet écart entre le champ du discours et l'intérêt politique souligne la complexité de l'adhésion sociale aux normes défendues par les autorités politiques. On peut ainsi concevoir une autonomisation du discours qui remet en cause les schémas traditionnels d'explication sur les sociétés coloniales.

Pierre-Éric FAGEOL

Université de La Réunion CRESOI-Centre de recherches sur les sociétés de l'océan Indien 